\title{
Correlating optic nerve sheath diameter with opening intracranial pressure in pediatric traumatic brain injury
}

\author{
Adam M.H. Young ', Mathew R. Guilfoyle', Joseph Donnelly', Daniel Scoffings ${ }^{2}$, Helen Fernandes ${ }^{1}$, Mathew Garnett ${ }^{1}$, \\ Shruti Agrawal ${ }^{3}$ and Peter J. Hutchinson ${ }^{1}$
}

\begin{abstract}
INTRODUCTION: The use of clinical markers to predict intracranial pressure (ICP) is desirable as a first-line measure to assist in decision making as to whether invasive monitoring is required. Correlations between ICP and optic nerve sheath diameter (ONSD) using CT and MRI have been observed in adult populations. However, data on this modality in children is less well documented.
\end{abstract}

METHODS: ONSD was measured by independent observers and correlated with opening ICP at insertion of invasive monitoring probes in pediatric traumatic brain injury patients admitted to Addenbrookes Hospital between January 2009 and December 2013.

RESULTS: Thirty-six patients with a mean age of $8.2 \mathrm{y}$ were admitted to the Pediatric Intensive Care Unit (PICU) with a traumatic head injury and required invasive neurosurgical monitoring. The median ICP was $18 \pm 10 \mathrm{mmHg}$ (median \pm IQR), the median right ONSD was $5.6 \pm 2.5 \mathrm{~mm}$ and the left was $5.9 \pm 3.2 \mathrm{~mm}$. The Intraclass correlation between observers was $0.91(P<0.0001)$. The correlation of mean ONSD and max ONSD with ICP was $0.712(P<0.0001)$ and $0.713(P<0.0001)$, respectively. Area under ROC curve for both mean and max ONSD is 0.85 (95\% Cl: 0.73-0.98).

CONCLUSION: Where pediatric patients present with an ONSD of over $6.1 \mathrm{~mm}$ following a traumatic brain injury (TBI), ICP monitoring should be implemented.

$\mathrm{n}$ adults with severe traumatic brain injury (TBI), increased intracranial pressure (ICP) is associated with secondary brain injury and is a strong predictor of poor neurologic outcome in this population $(1,2)$. In children, it is presumed that this is equally as detrimental however, due to the anatomical differences in both cranial rigidity and subdural space, the resulting impact on intracranial compliance is uncertain. Ultimately, as with adults, in children elevated ICP in the context of traumatic brain injury can result in significant secondary ischemic injury by limiting cerebral blood flow and as a result cerebral oxygen $(3,4)$.
The gold standard for monitoring intracranial pressure is by using an invasive ICP wire or external ventricular drain. Indications for the application of this device are based on clinical observations. As a guide, (i) patients who present with a post-resuscitation Glasgow coma score (GCS) of 8 or less after resuscitation, in the presence of an abnormal CT head scan or (ii) with a normal $\mathrm{CT}$ head but have $>2$ risk factors $(\mathrm{SBP}<90$ $\mathrm{mmHg}$, decorticate or decerebrate posturing); (iii) patients with multiple injuries with an altered level of consciousness; (iv) patients with reduced GCS subsequent to removal of an intracranial mass are recommended to have invasive monitoring $(5,6)$. The difficulty lies in patients with GCS between 9 and 12 who may benefit from aggressive medical therapy which can only be accurately guided by invasive monitoring nevertheless, the decision to undertake these procedures can often be difficult.

Unfortunately, the traditional features that are used on computerized tomography (CT) head imaging (midline shift, basal cistern, and sulcal effacement) do not reliably predict elevated ICP $(7,8)$. ICP may be highly dynamic, almost instantly changing its value from normal baseline to grossly elevated (9).

The optic nerve sheath is continuous with the meninges of the central nervous system and is encased with the subarachnoid membrane. Cerebrospinal fluid (CSF), located in the subarachnoid space, accumulates in the optic nerve sheath thereby widening its diameter in the setting of increased ICP and limited intracranial compliance $(10,11)$. Human studies have shown that this phenomenon occurs within minutes of acute changes in ICP (10-12).

Ultrasonography of ONSD in TBI patients has been shown to correlate with increased ICP (13-16) and systemic reviews have supported this observation $(17,18)$. Although reliability of ONSD measurements with ultrasound has been established, an element of technical expertise is required to obtain adequate images which likely limits its ubiquitous clinical utility $(19,20)$. While both MRI and CT measurements have been validated in adults (21), there is limited evidence in children. Here, we review the referred CT scans of pediatric patients with

'Division of Academic Neurosurgery, Department of Clinical Neurosciences, Addenbrooke's Hospital, University of Cambridge, Cambridge, UK; ${ }^{2}$ Department of Neuroradiology, Addenbrooke's Hospital, University of Cambridge, Cambridge, UK; ${ }^{3}$ Department of Pediatric Intensive Care, Addenbrooke's Hospital, University of Cambridge, Cambridge, UK. Correspondence: Adam M.H. Young (ay276@cam.ac.uk)

Received 19 February 2016; accepted 16 June 2016; advance online publication 9 November 2016. doi:10.1038/pr.2016.165 


\section{Articles | Young et al.}

traumatic brain injury and correlate the ONSD with opening ICP at time of surgery.

\section{RESULTS}

\section{Demographics}

Thirty-six patients with a mean age of 8.2 y were admitted to the PICU with a traumatic head injury and required invasive neurosurgical monitoring. The baseline clinical features are outlined in Table 1.

The Modified Marshall scores ranged from 1-6 with a mode of 2. Specific CT features included subarachnoid hemorrhage in $69 \%$ of patients and subdural hemorrhage and extradural hemorrhage in 11 and 25\% respectively. Of the extra-axial hemorrhages identified, $73 \%$ were evacuated. Petechial hemorrhages were observed in 33\% of the cohort and obliteration of the basal cisterns in 36\% if the cohort. Midline shift was observed in $39 \%$ (Table 1). Mortality was $17 \%$ both at $14 \mathrm{~d}$ and at 6 mo. Of those who survived $33 \%$ had a good recovery $5,39 \%$ had moderate disability, and $11 \%$ had a poor but survivable outcome ( $8 \%$ severe disability, $3 \%$ vegetative state) according to the Glasgow outcome scale.

Table 1. Patient demographics used to calculate outcome scores

\begin{tabular}{lc}
\hline & $\begin{array}{c}\text { Patients } \\
(n=36)\end{array}$ \\
\hline Age, mean \pm SD & $8.2 \pm 7.1$ \\
Male (\%) & $24(66)$ \\
Admission Glasgow coma score, median (range) & $8(3-12)$ \\
Motor score & $4(1-4)$ \\
Pupils & \\
Reactive (\%) & $29(81)$ \\
Fixed unilaterally (\%) & $4(11)$ \\
Fixed bilaterally (\%) & $3(8)$ \\
Prehospital hypoxia & $8(22)$ \\
Pre-hospital Hypotension & $12(33)$ \\
Modified Marshall score, (mode) & 2 \\
Subarachnoid hemorrhage on CT (\%) & $25(69)$ \\
Epidural mass (\%) & $13(36)$ \\
Petechial hemorrhages (\%) & $12(33)$ \\
Obilteration of basal cisterns (\%) & $13(36)$ \\
Mid-line shift (\%) & $14(39)$ \\
Surgical intervention & $12(33)$ \\
External ventricular drain & $14(39)$ \\
Hematoma evacuation & $3(8)$ \\
Decompression & $1(3)$ \\
Goodcome & $4(11)$ \\
Moderate disability & $12(3)$ \\
Severe disability & \\
\hline
\end{tabular}

\section{ONSD Measurements}

The inter-rater agreement for ONSD measurements as defined by single-score intraclass correlation was 0.91 (95\% CI: 0.850.94; $P<0.0001$; Figure 1). The median ICP was $18 \pm 10 \mathrm{mmHg}$ (median \pm IQR), the median right ONSD was $5.6 \pm 2.5 \mathrm{~mm}$ and the left was $5.9 \pm 3.2 \mathrm{~mm}$. Both mean ONSD and maximum ONSD were significantly correlated with initial ICP ( $r=0.712, P<0.0001$ and $r=0.713, P<0.0001$, respectively). Linear regression of ONSD against ICP, age, and sex found that only ICP was significantly associated with ONSD $(P<0.0001)$; there was no significant relationship between ONSD and age $(P=0.970)$ or sex $(P=0.340)$.

Area under Receiver-operating characteristic (ROC) curve for both mean and max ONSD was 0.85 (95\% CI: 0.71-1.00) (Figure 2).

The optimal threshold of mean ONSD identified by maximizing the J statistic was $6.1 \mathrm{~mm}$ (sensitivity $77 \%$, specificity $91 \%$ ) and the optimal threshold of maximum ONSD was $6.3 \mathrm{~mm}$

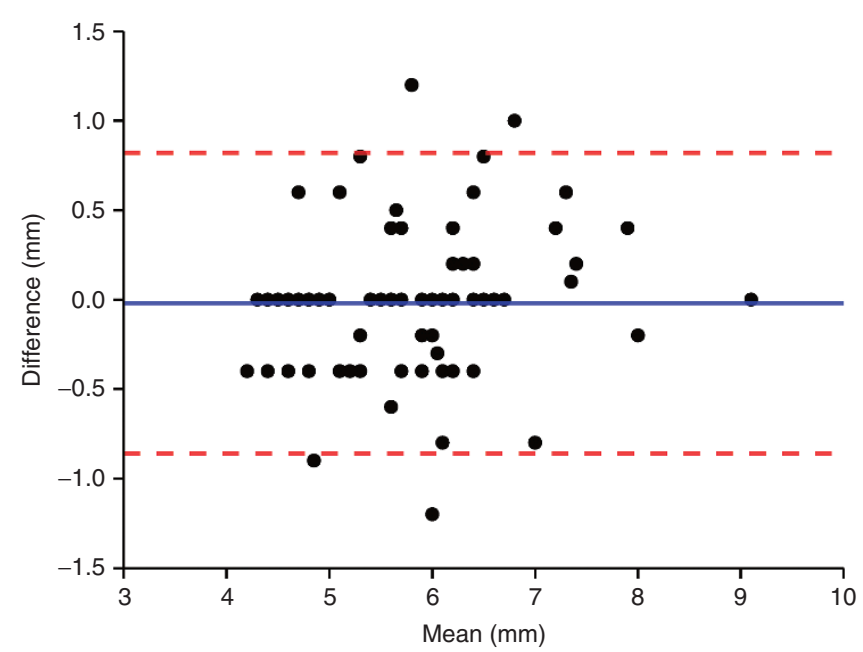

Figure 1. Bland-Altman plot demonstrating the intraclass correlation between observers was $0.91(P<0.0001)$.

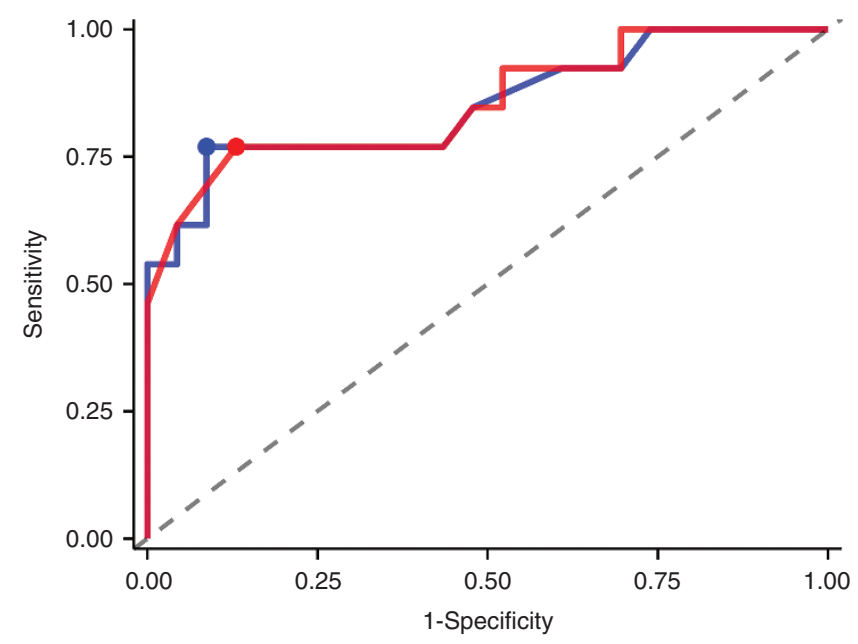

Figure 2. Area under ROC curve for both mean (blue) and max (red) optic nerve sheath diameter is $0.85(95 \% \mathrm{Cl}: 0.71-1.00)$. 
(sensitivity $77 \%$, specificity $87 \%$ ). The threshold for $100 \%$ sensitivity for mean ONSD was $4.9 \mathrm{~mm}$ (specificity 26\%) and for maximum ONSD was $5.2 \mathrm{~mm}$ (specificity $30 \%$; Figure 3 ).

A linear regression of ICP against ONSD together with GCS (total or motor score only), pupillary reactivity, and imaging severity (Marshall score or separate features of basal cistern obliteration, midline shift, petechial hemorrhage, subarachnoid hemorrhage, and extra-axial hematoma) showed that only GCS motor score of $2(P=0.019)$ and ONSD $(P<0.0001)$ were independently associated with ICP $(P>0.1$ for all other factors).

\section{DISCUSSION}

The use of invasive monitoring in children with severe traumatic brain injury is gaining momentum as a protective measure against secondary injury. In adults, it is regularly employed to provide information on brain pressures, oxygenation, and cellular metabolism. Nevertheless, in children, a decision to use invasive monitoring devices should never be taken lightly. Here, we describe data from a retrospective cohort which demonstrates a linear correlation between ONSD on the referring $\mathrm{CT}$ with opening ICP in critical pediatric patients with traumatic brain injury. ONSD measurements showed excellent agreement between raters. ONSD also showed excellent discriminative ability to predict patients with intracranial hypertension.

The relationship between ONSD and ICP is based on the sheath's continuity with the subarachnoid membrane (11). ICP rises during periods of limited intracranial compensatory reserve and compliance (11), during which CSF is redistributed to the optic nerve sheath and displaces the sheath.

The approximately linear relationship between ONSD and ICP demonstrated in our study is similar to previously published studies in adult patients using CT (22), ultrasound $(15,16,23)$, and MRI (24). The ability for these techniques to discriminate between elevated and acceptable ICP was dependent on the threshold for ONSD cut-off. Using ultrasound to

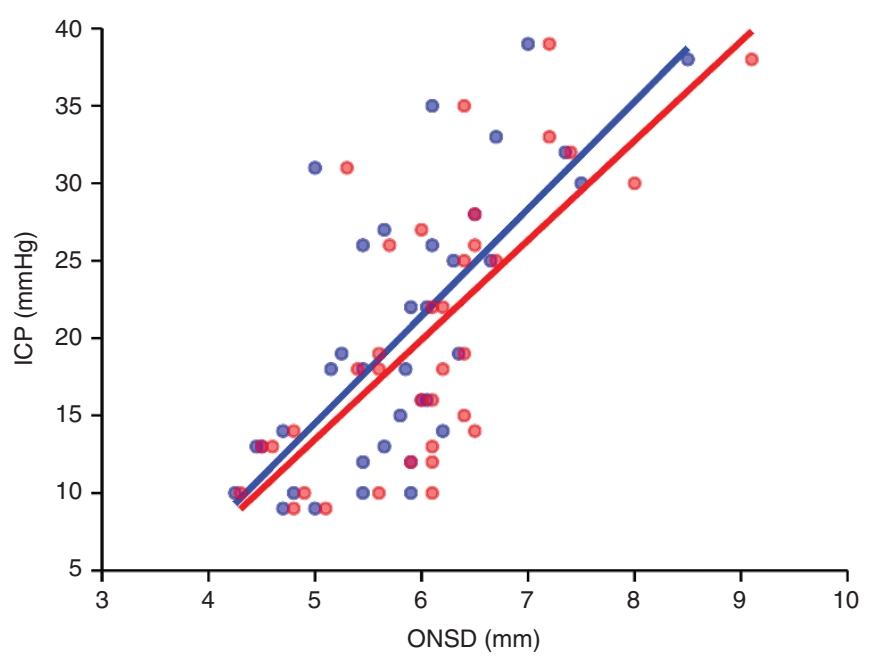

Figure 3. Correlations between mean ONSD (blue) and maximum ONSD (red) with opening intracranial pressure. ONSD, optic nerve sheath diameter. achieve a sensitivity of $95 \%$ and a specificity of $79 \%$, the threshold was set at $5.86 \mathrm{~mm}$ in adults (24). In MRI, an improved sensitivity of $90 \%$ and a specificity of $92 \%$ was achieved with a recued threshold of $5.82 \mathrm{~mm}$ (24). Finally, CT in adults demonstrated the poorest specificity. Setting the threshold at $6.0 \mathrm{~mm}$, a sensitivity of $97 \%$ was achieved with a specificity of $42 \%$ affording a significantly increased risk of false positives (22). Interesting in a pediatric population, both a high specificity and sensitivity was achieved. Although the threshold of $4.9 \mathrm{~mm}$ cannot be compared to that selected in adults, previous studies in normal children have declared that a limits of $4.00 \mathrm{~mm}$ in infants ( $<1 \mathrm{y}$ old) and $4.50 \mathrm{~mm}$ for older children should be treated as normal.

The improved sensitivity in children compared to adults could be associated with natural anatomy of the CSF spaces in these patients (25). In children, the brain parenchyma is intimate with the cranial vault and only as the brain ages through late adolescence and early adulthood does the formation of deeper sulci allow for greater anatomical variability among patients (25). As such, the pediatric brain is more sensitive to changes in intracranial pressure and measurements of noninvasive intracranial pressure can be reproduced with high sensitivity and specificity.

Additionally, ONSD estimation of ICP is highly dependent on normal intracranial flow dynamics (22) to allow for adequate displacement of the sheath. Following a severe TBI, CSF flow dynamics are likely to become altered, particularly in the presence of mass lesions of extra-axial hemorrhage or obstructive hydrocephalus. Again, the anatomical differences between adult and pediatric cohorts may prevent the gross variability observed in adults consequently improving the accuracy to the technique in this cohort.

Finally, as ONSD appears to be superior in predicting pathological ICP over the historical features of cisternal effacement, sulcal effacement, ventricular compression, and cerebral herniation in this pediatric cohort, it is argued that ONSD could be utilized as a predictor of intracranial hypertension to guide as a screening tool for those who may require more continuous invasive intracranial monitoring. Although our findings do not suggest a cut-off that can be applied universally across pediatric cohorts without further evaluation we have identified that where an ONSD diameter of $4.9 \mathrm{~mm}$ is observed, a raised ICP is unlikely. This is particularly useful in pediatric cohorts where the skull is thin and insertion of ICP monitors carries a higher risk of disrupting normal tissues (25). Furthermore, it can also provide reassurance in patients who are not suitable for invasive monitoring coagulopathy and hypoxemic-ischemic brain injury post-cardiac arrest with concomitant use of antiplatelet or anticoagulant therapies.

The inter-observer correlation for ONSD in the present study was high. Nonetheless, even small variations between observers become important when close to the set thresholds that are identified as predictive of raised ICP. Future studies in larger cohorts are needed to examine the intra- and inter-observer variability of ONSD measurement and corresponding proportion of patients that are classified differently by independent 


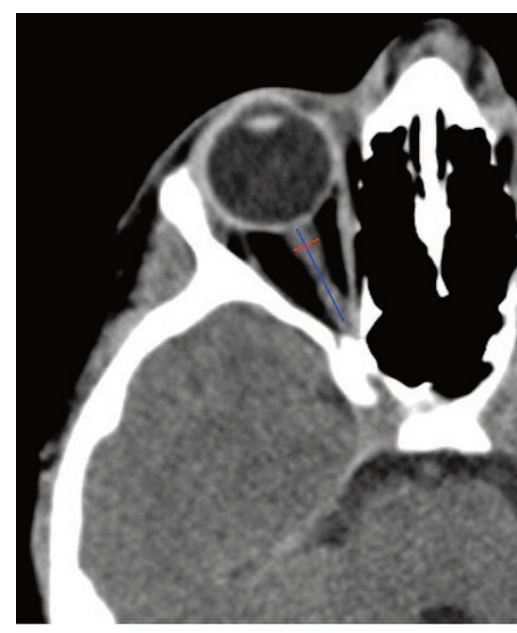

Figure 4. CT scan of patient with traumatic brain injury demonstrating the measurement of optic nerve sheath diameter.

assessors. Simple techniques to minimize variation and misclassification might include averaging two or more repeated measurements by the same or alternative assessors. However, despite any shortcomings ONSD may still be superior to traditional indicators of raised ICP based on CT imaging (e.g., basal cistern effacement) that are typically qualitative and subject to substantial inter-observer variation (26).

An important limitation of this preliminary report is the small sample size. We did not observe a relationship between ONSD and age but this remains an important area for further study as application of this technique in pediatric TBI is crucially dependent on understanding the normal range of ONSD through child development. The patients in the present study are inherently highly selected, and therefore, the positive or negative predictive value of the thresholds reported here cannot be immediately extrapolated to the wider pediatric TBI population. Again, larger comprehensive multicenter population studies are needed to confirm the utility and validity of ONSD measurements.

The measurement of ONSD in TBI has been most regularly assessed using ultrasound (13-16). Although correlations between ICP and ONSD using this technique are strong, it is widely recognized that, an element of technical expertise is required to obtain adequate images which likely limits its ubiquitous clinical utility $(19,20)$. As such, the use of CT studies to limit variability in this population may be adventageous.

\section{Conclusion}

ONSD on CT can be used as a predictor of raised intracranial pressure in pediatric head injury. Where pediatric patients present with an ONSD of over $6.1 \mathrm{~mm}$ following a TBI, ICP monitoring should be implemented. For values lower than $6.1 \mathrm{~mm}$, there remains a considerable risk of a patient having a raised ICP and as such treatment and management should be implemented using a hollistic clinical picture.

\section{METHODS \\ Patients}

The data in this study were collected retrospectively from data records of pediatric severe traumatic brain injury patients admitted to Addenbrookes Hospital Pediatric Intensive Care Unit (PICU) between January 2009 and December 2013. The insertion of an intracranial monitoring device is part of routine clinical practice and as such did not require ethical approval. The data is routinely collected for clinical purposes and guides the management of patients. The analysis of data within this study for the purposes of service evaluation was approved by the Cambridge University Hospital NHS Trust, Audit and Service Evaluation Department (Ref: 2143) and did not require ethical approval nor patient consent.

Inclusion criteria were as follows: (i) Confirmed traumatic brain injury i.e. structural abnormality, confirmed on CT or MRI, (ii) severe injury or failure to demonstrate significant early clinical improvement (i.e., poor neurology on sedation hold) or an injury which required close conservative management or neurological monitoring in PICU, (iii) patients requiring invasive monitoring of ICP and ABP.

Patients were managed according to current TBI guidelines (6). Interventions were aimed at keeping ICP $<20 \mathrm{~mm} \mathrm{Hg}$ using a tiered treatment protocol of positioning, sedation, muscle paralysis, moderate hyperventilation, ventriculostomy, osmotic agents, and induced hypothermia. Clinical outcome was determined using the Glasgow Outcome Scale.

\section{Data Acquisition}

Patient demographics and 6-mo follow-up data were obtained from electronic records. Serum marker levels were extracted from admission blood tests. CT findings were obtained from referring images to the Department of Neurosurgery, Addenbrookes Hospital. Information on admitting clinical features (e.g., Glasgow Coma Scale (GCS), hypotension and hypoxia) were obtained from the PICU discharge summary.

\section{Optic Nerve Sheath Measurements}

Independently, two investigators (A.Y., D.S.), not involved with data collection and blinded to the patient's condition, measured bilateral ONSD from the initial admission CT head. All CT scans were performed with a 16 section multi-detector row CT scanner (Somatom Sensation 16 scanner, Siemens, Germany). Images were acquired in spiral mode with a section thickness of $1 \mathrm{~mm}$. Each observer scrolled through the images to identify the level demonstrating a visual estimation of the largest diameter. Images were displayed at a window level of $30 \mathrm{HU}$ and window width of $74 \mathrm{HU}$. The image was then magnified and the ONSD on each nerve was measured using electronic calipers, once per side. The ONSD was measured as previously described by Legrand et al. (27). Briefly, the ONSD was measured $3 \mathrm{~mm}$ behind the insertion of the optic nerve into the globe, perpendicular to the long axis of the optic nerve using a digital viewer (Centricity PACS, General Electric Healthcare, Chicago, IL) with an electronic caliper (Figure 4). We calculated the mean ONSD between sides, which was used for the analysis.

\section{Statistical Analysis}

All statistical analysis was performed in $\mathrm{R}$ (v.3.2.0) significance was set at $P<0.05$. Inter-rater reliability of ONSD measurement between investigators was quantified with the single-score intraclass correlation coefficient; agreement between investigators was assessed using a Bland-Altman plot (28). The correlation between ONSD and ICP was evaluated with Pearson's coefficient. The performance of ONSD in predicting high opening intracranial pressure was examined by classifying the initial ICP following insertion of monitoring as high $(\geq 20 \mathrm{mmHg})$ or low $(<20 \mathrm{mmHg})$. Receiver-operating characteristic curves of patients mean and maximum ONSD against the dichotomized initial ICP were then constructed and the area-underthe-curve (AUC) was determined. Generally, AUC $>0.8$ is considered to indicate "good" performance. The optimal threshold of ONSD balancing sensitivity and specificity for predicting high ICP was calculated by maximising Youden's J statistic (29); the threshold for 100\% sensitivity was also noted. 


\section{STATEMENT OF FINANCIAL SUPPORT}

We gratefully acknowledge financial support as follows. Research support: the Medical Research Council (MRC, Grant Nos. G0600986 ID79068 and G1002277 ID98489) and the National Institute for Health Research Biomedical Research Centre (NIHR BRC) Cambridge (Neuroscience Theme; Brain Injury and Repair Theme). Authors' support: P.J.H.-NIHR Research Professorship, Academy of Medical Sciences/Health Foundation Senior Surgical Scientist Fellowship and NIHR Cambridge BRC. J.D. is supported by a Woolf Fisher Scholarship. MC- NIHR BRC.

Disclosure: The authors have no conflicts of interest relevant to this article to disclose

\section{REFERENCES}

1. Myburgh JA, Cooper DJ, Finfer SR, et al.; Australasian Traumatic Brain Injury Study (ATBIS) Investigators for the Australian; New Zealand Intensive Care Society Clinical Trials Group. Epidemiology and 12-month outcomes from traumatic brain injury in australia and new zealand. J Trauma 2008;64:854-62.

2. Juul N, Morris GF, Marshall SB, Marshall LF. Intracranial hypertension and cerebral perfusion pressure: influence on neurological deterioration and outcome in severe head injury. The Executive Committee of the International Selfotel Trial. J Neurosurg 2000;92:1-6.

3. Balestreri M, Czosnyka M, Hutchinson P, et al. Impact of intracranial pressure and cerebral perfusion pressure on severe disability and mortality after head injury. Neurocrit Care 2006;4:8-13.

4. Chesnut RM, Marshall LF, Klauber MR, et al. The role of secondary brain injury in determining outcome from severe head injury. J Trauma 1993;34:216-22.

5. Brain Trauma Foundation, American Association of Neurological Surgeons, Congress of Neurological Surgeons, Joint Section on Neurotrauma and Critical Care (AANS/CNS) et al. Guidelines for the management of severe traumatic brain injury. I. Blood pressure and oxygenation. J Neurotrauma 2007;24 Suppl 1:S7-S13

6. Kochanek PM, Carney N, Adelson PD, et al. Guidelines for the acute medical management of severe traumatic brain injury in infants, children, and adolescents--second edition. Pediatr Crit Care Med 2012;13 Suppl 1:S1-82.

7. Hiler M, Czosnyka M, Hutchinson P, et al. Predictive value of initial computerized tomography scan, intracranial pressure, and state of autoregulation in patients with traumatic brain injury. J Neurosurg 2006;104:731-7.

8. Miller MT, Pasquale M, Kurek S, et al. Initial head computed tomographic scan characteristics have a linear relationship with initial intracranial pressure after trauma. J Trauma 2004;56:967-72; discussion 972-3.

9. Czosnyka M, Pickard JD. Monitoring and interpretation of intracranial pressure. J Neurol Neurosurg Psychiatry 2004;75:813-21.

10. Launey Y, Nesseler N, Le Maguet P, Mallédant Y, Seguin P. Effect of osmotherapy on optic nerve sheath diameter in patients with increased intracranial pressure. J Neurotrauma 2014;31:984-8.

11. Helmke K, Hansen HC. Fundamentals of transorbital sonographic evaluation of optic nerve sheath expansion under intracranial hypertension. I. Experimental study. Pediatr Radiol 1996;26:701-5.

12. Hansen HC, Helmke K. Validation of the optic nerve sheath response to changing cerebrospinal fluid pressure: ultrasound findings during intrathecal infusion tests. J Neurosurg 1997;87:34-40.
13. Tayal VS, Neulander M, Norton HJ, Foster T, Saunders T, Blaivas M. Emergency department sonographic measurement of optic nerve sheath diameter to detect findings of increased intracranial pressure in adult head injury patients. Ann Emerg Med 2007;49:508-14.

14. Rajajee V, Vanaman M, Fletcher JJ, Jacobs TL. Optic nerve ultrasound for the detection of raised intracranial pressure. Neurocrit Care 2011;15: 506-15.

15. Geeraerts T, Launey Y, Martin L, et al. Ultrasonography of the optic nerve sheath may be useful for detecting raised intracranial pressure after severe brain injury. Intensive Care Med 2007;33:1704-11.

16. Geeraerts T, Merceron S, Benhamou D, Vigué B, Duranteau J. Noninvasive assessment of intracranial pressure using ocular sonography in neurocritical care patients. Intensive Care Med 2008;34:2062-7.

17. Moretti R, Pizzi B. Ultrasonography of the optic nerve in neurocritically ill patients. Acta Anaesthesiol Scand 2011;55:644-52.

18. Dubourg J, Javouhey E, Geeraerts T, Messerer M, Kassai B. Ultrasonography of optic nerve sheath diameter for detection of raised intracranial pressure: a systematic review and meta-analysis. Intensive Care Med 2011;37:1059-68.

19. Tsung JW, Blaivas M, Cooper A, Levick NR. A rapid noninvasive method of detecting elevated intracranial pressure using bedside ocular ultrasound: application to 3 cases of head trauma in the pediatric emergency department. Pediatr Emerg Care 2005;21:94-8.

20. Bhardwaj G, Chowdhury V, Jacobs MB, Moran KT, Martin FJ, Coroneo MT. A systematic review of the diagnostic accuracy of ocular signs in pediatric abusive head trauma. Ophthalmology 2010;117:983-992.e17.

21. Kalantari H, Jaiswal R, Bruck I, et al. Correlation of optic nerve sheath diameter measurements by computed tomography and magnetic resonance imaging. Am J Emerg Med 2013;31:1595-7.

22. Sekhon MS, Griesdale DE, Robba C, et al. Optic nerve sheath diameter on computed tomography is correlated with simultaneously measured intracranial pressure in patients with severe traumatic brain injury. Intensive Care Med 2014;40:1267-74.

23. Kimberly HH, Noble VE. Using MRI of the optic nerve sheath to detect elevated intracranial pressure. Crit Care 2008;12:181.

24. Geeraerts T, Newcombe VF, Coles JP, et al. Use of T2-weighted magnetic resonance imaging of the optic nerve sheath to detect raised intracranial pressure. Crit Care 2008;12:R114.

25. Dennis EL, Ellis MU, Marion SD, et al. Callosal Function in Pediatric Traumatic Brain Injury Linked to Disrupted White Matter Integrity. J Neurosci 2015;35:10202-11.

26. Harrison DA, Prabhu G, Grieve R, et al. Risk Adjustment In Neurocritical care (RAIN)-prospective validation of risk prediction models for adult patients with acute traumatic brain injury to use to evaluate the optimum location and comparative costs of neurocritical care: a cohort study. Health Technol Assess 2013;17:vii-viii, 1-350.

27. Legrand A, Jeanjean P, Delanghe F, Peltier J, Lecat B, Dupont H. Estimation of optic nerve sheath diameter on an initial brain computed tomography scan can contribute prognostic information in traumatic brain injury patients. Crit Care 2013;17:R61.

28. Bland JM, Altman DG. Statistical methods for assessing agreement between two methods of clinical measurement. Lancet 1986;1:307-10.

29. Youden WJ. Index for rating diagnostic tests. Cancer 1950;3:32-5. 